\title{
Citation analysis of the scientific publications of Britton Chance in ISI citation indexes
}

\author{
Lin Z. $\mathrm{Li}^{*, \dagger}{ }^{*}$, Loet Leydesdorff ${ }^{\sharp}$, Shoko Nioka ${ }^{\dagger}$, \\ Nannan Sun ${ }^{*, \dagger} \S$ and Eugene Garfield ${ }^{\mathbb{T}}$ \\ *Department of Radiology, Perelman School of Medicine \\ University of Pennsylvania, Philadelphia, PA 19104, USA \\ ${ }^{\dagger}$ Britton Chance Laboratory of Redox Imaging, \\ Johnson Research Foundation \\ Department of Biochemistry and Biophysics, \\ Perelman School of Medicine \\ University of Pennsylvania, Philadelphia, PA 19104, USA \\ ¿niversity of Amsterdam \\ Amsterdam School of Communications Research (ASCoR), Netherlands \\ $\S_{\text {Britton Chance Center for Biomedical Photonics }}$ \\ Wuhan National Laboratory for Optoelectronics Huazhong \\ University of Science and Technology \\ Wuhan 430074, P. R. China \\ IInstitute for Scientific Information - Thomson Reuters \\ Philadelphia, PA 19130, USA \\ "linli@mail.med.upenn.edu
}

Received 4 March 2014

Accepted 4 March 2014

Published 2 April 2014

\begin{abstract}
Britton Chance was a pioneer in many scientific fields such as enzymatic reaction kinetics, bioenergetics, metabolism, in vivo NMR, and biophotonics. As an engineer, physical chemist, physicist, physiologist, biophysicist, biochemist, innovator and educator, he had worked in diversified fields over extended periods between 1926 until his death in 2010, at the age of 97 . In order to illustrate his scientific career and great impact on research from a new perspective, we employ scientometric analysis tools to analyze the publications of Britton Chance with data downloaded from the ISI Citation Indexes in April 2013. We included articles, reviews and proceeding papers but excluded meeting abstracts. In total, we obtained 1023 publication records with 1236 authors in 266 journals with 17,114 citations from 1945 to 2013 . We show the annual publications and citations that Britton Chance received from 1945 to 2013, and generate HistCite maps on the basis of the global citations (GCS) and local (self) citations (LCS) to show the citation relationships among the top-30 publications of Britton Chance. Metabolism and the
\end{abstract}

This is an Open Access article published by World Scientific Publishing Company. It is distributed under the terms of the Creative Commons Attribution 3.0 (CC-BY) License. Further distribution of this work is permitted, provided the original work is properly cited. 


\section{Z. Li et al.}

development of physical methods to probe it appear to be the connecting thread of the lifelong research of Britton Chance. Furthermore, we generate the journal map and co-authorship map to show the broad scope of research topics and collaborators and the high impacts of the scientific oeuvre of Britton Chance ranging from physics, engineering, chemistry and biology to medicine.

Keywords: Scientometric analysis; impact; HistCite; citation tree; metabolism; mitochondria.

\section{Introduction to Britton Chance}

Britton Chance (1913-2010), one of the most outstanding scientists in the world in the 20th century, had been a legendary figure in the history of science. He was famous for his enthusiasm in sailing due to his family tradition, which brought him the rare experience of catching a big, over $400 \mathrm{lb}$ blue marlin in Caribbean Sea, an Olympic Gold Medal for $5.5 \mathrm{~m}$ boat sailing in 1952, and a couple of world championships of sailing in 1960s. However, his talent, enthusiasm and fame were far more distinguished in science. As a teenager, he invented an optoelectric device for ship autosteering, which was later tested satisfactorily for large commercial ships such as the Texas Sun and New England Star in 1930s. Along with these testing trips, he was admitted to the Trinity College of Cambridge University in late 1930s under the mentorship of Glenn Millikan, the son of the Nobel Laureate Robert A. Millikan, who measured the charge of the electron. Eventually, he graduated with two PhDs, one in Physical Chemistry from the University of Pennsylvania in 1940, and one in Physiology from the University of Cambridge in 1942. His $\mathrm{PhD}$ thesis was on developing a fast mini stop-flow device for measuring the kinetics of enzymatic reactions. With such a device, he achieved the first experimental, quantitative demonstration of the existence of Michaelis-Menten enzyme-substrate complex in early 1940s. He was also highly skillful with circuits and electronics. From 1941-1946, he was recruited to the Radiation Lab in MIT for developing advanced radar systems including anti-aircraft radar SCR584 and airborne radar bomber sight used for fighting against Nazi during World War II. He quickly rose to be a member of Steering Committee of the Radiation Lab, leading a large group of physicists with over 200 people. His group taught Presper Eckert how to make key circuits and overcome some technical problems for ENIAC, the world 1st general purpose electronic digital computer at the University of Pennsylvania in 1946. From 1940s till 2010 except a couple of short term leaves, he stayed in the
Johnson Research Foundation, Department of Biochemistry and Biophysics at the University of Pennsylvania for seven decades. From 2006 to 2010, he spent half a year annually in research institutions in Asian countries and regions such as Singapore, Mainland China and Taiwan to conduct research and education, helping the development of biophotonics in those places.

His eight decades of scientific research (19262010) culminated into numerous scientific discoveries and technological inventions. He had advanced many frontier research fields at the time with both the development of new methods/devices and their applications to key biological questions. Apart from the aforementioned enzyme kinetics studies with his mini stop-flow device, in 1950s, Chance invented the dual-wavelength spectrophotometer which has been widely used for studying turbid biological samples around the world until today. He applied this instrument to extensive investigations on the electron transport in mitochondrial respiration, redox cofactors and metabolic control mechanisms. In 1960s, he first discovered the electronic tunneling process in biological systems. In 1970s, he identified hydrogen peroxide released by the respiratory chain in mitochondria. From 1970s to early 1980s, he developed the 3D cryogenic redox scanner for imaging tissue redox state and its heterogeneity at submillimeter resolution. In the 1970-1980s, he was also a key player in developing in vivo ${ }^{31} \mathrm{P}-\mathrm{NMR}$ spectroscopy for bioenergetics studies and using X-ray spectroscopy to elucidate the structure-function relation of biomolecules. Since late 1980s, Prof Chance and his collaborators had founded the field of biophotonics by developing in vivo NIR spectroscopy and imaging methods, mainly based on hemoglobin absorption, time-resolved spectroscopy, photon diffusion, etc. These methods have been widely applied in both laboratory and clinic studies for the measurement of blood oxygenation, volume, and flow, brain activities, muscle functions and cancer detections and diagnosis. The new research directions he developed 
during the last decade of his life includes developing novel molecular beacons for cancer diagnosis and therapy, predicting cancer aggressiveness by redox scanning, and developing an oral optical metabolometer to detect nutritional status in human subjects. The latter two directions completed the circle of his scientific research since 1950s by utilizing intrinsic optical signals from mitochondria.

Among many honors and awards ${ }^{1}$ he has received are the memberships of national academies of sciences from six countries including United States, Italy, United Kingdom, Sweden, Germany and Argentina; over 10 honorary $\mathrm{PhD}$ and MD degrees; US National Medal of Sciences (1974); The Gold Medal for Distinguished Service to Medicine, College of Physicians, USA (1987); The Gold Medal of the Society of Magnetic Resonance in Medicine, USA (1988); The Benjamin Franklin Medal for Distinguished Achievement in the Sciences, American Philosophical Society (1990); The Christopher Columbus Discovery Award in Biomedical Research, National Institutes of Health, USA (1992); The International Society for Optical Engineering (SPIE) Lifetime Achievement Award (2005); The Gold Medal of American Roentgen Ray Society (2006); Distinguished Achievement Award of American Aging Association (2006). Due to his tireless effort in promoting scientific exchange and collaboration between the East and the West, Chance received the Friendship Award from the State Administration of Foreign Experts Affairs of China in 2008. He was further recognized in 2009 with the International Science and Technology Cooperation Award, which is China's highest national honor given to foreign scientists. In 2013, Chance was inducted into the Innovators Walk of Fame at the University City Science Center, Philadelphia.

\section{Scientometric Analysis}

To commemorate a great scholar and carry on his/ her legacy to future generations, scientometric analysis can be used in addition to anecdotal stories. Scientometric analyses have been utilized to evaluate the achievements of scholars, ${ }^{2}$ and usually these scholars have a focus within a specific field. Here we employed several tools to evaluate the scientific contribution of Britton Chance whose career spanning multiple disciplines. Our analysis was based on a dataset downloaded from the ISI Citation Indexes in April, 2013. Articles, reviews and proceeding papers were included with no meeting abstracts. We obtained 1023 publication records in total from 1945 to 2013 including 1236 authors in 266 journals with 17,114 citations.

Publication periods. We try to correlate the annual publication profile of Chance with his research activities. In these 67 years between 1945 and 2012 (age 32-97), average of 15.3 papers were published with 255 citations annually. Note that these numbers underestimate his published manuscripts, because many of his manuscripts were published in places not indexed by ISI Citation Indexes yet. His publications during his graduate studies were not covered by ISI. Moreover, during the time before World War II ended in 1945, he worked in the area of electronic engineering in a secret mission at Radiation Laboratory, thus, most of his works were not published in academic journals.

Figure 1 shows the annual number of published manuscripts from 1945-2012. At a glance, there are seven periods of Gaussian distributions over the whole time, and each one seems to represent roughly main subjects of his works. The publications of these periods went up and down, and we found that, for quite a few occasions, the publications of new ideas changed the research direction of Chance and boosted his publication records.

The first Gaussian period (1945-1956, peak at 1952) includes a number of applications of the micro stop-flow method to enzyme-substrate kinetics ${ }^{3-5}$ and the development of dual beam spectrometer ${ }^{6}$ enabling the study of turbid biological samples. With those apparatuses, Chance was active himself in several areas as well as he helped many other research works in various fields of physiology, chemistry, biochemistry, enzymology, etc. In 1947, Chance was the first to identify catalase compound I as an intermediate in the reaction of hydrogen peroxide and catalase. ${ }^{7}$ In 1955 , he published a landmark paper series with G.R. Williams on respiratory enzymes in oxidative phosphorylation. ${ }^{8-11}$ Chance finally set his life work into the mitochondrial biochemistry.

The second period (1957-1968) contains a peak at 1959 with the key publications of Chance's lifelong work on metabolic control mechanisms. ${ }^{12-17}$ The function of mitochondrial energy metabolism was well understood by the kinetic experiments of substrate and products relationships using the 


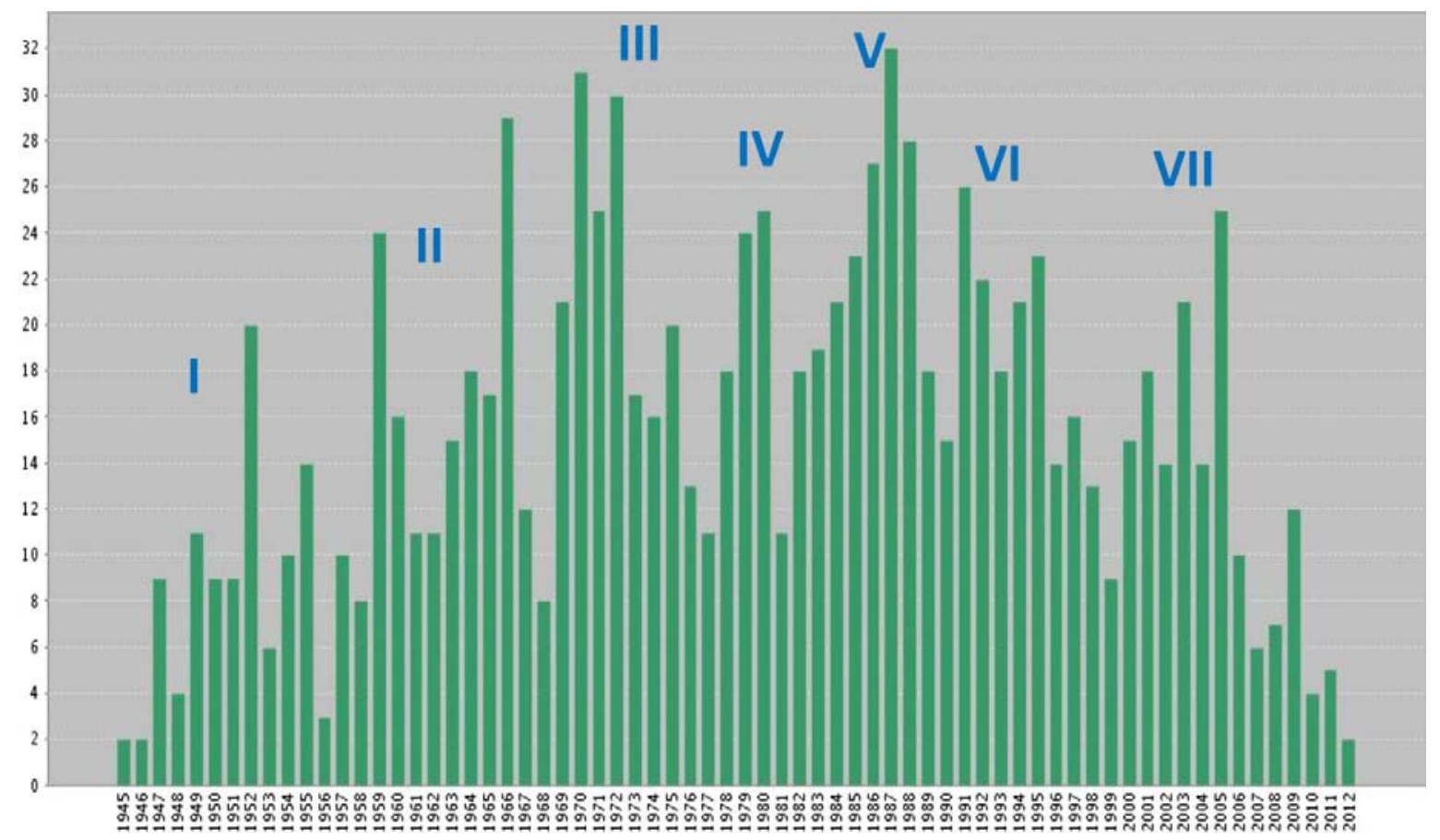

Fig. 1. The annual number of publications of Britton Chance from 1945-2012. Graph reproduced from ISI Citation Indexes (accessed in April, 2013 excluding meeting abstracts) with modifications. There were seven periods I-VII as described in the text.

double beam spectrometer. The peak at 1959 also came after the first observation of mitochondrial NADH fluorescence ${ }^{18}$ by him and Baltscheffsky in 1958 and coincided with the first observation of tissue NADH fluorescence ${ }^{19}$ by Chance and Jobsis in 1959. Chance et al. published in 1962 the first in vivo observation of $\mathrm{NAD}(\mathrm{P}) \mathrm{H}$ fluorescence and redox state from the brain and kidney. ${ }^{20-22}$ As we can see with his work on NADH fluorescence, Chance started with mitochondria followed by tissue and in vivo studies. This group of papers laid down a milestone for bioenergetics research and Chance's later work on the translational and clinical studies. From 1962-1968, Chance continued mitochondrial bioenergetics research on Complex I to IV with optical spectrometers and fluorometers of cytochromes and NADH. His publication peaked again in 1966 coinciding with another landmark discovery by him and DeVoult on the first experimental observation of quantum mechanical electron tunneling in biological system. ${ }^{23}$ The importance of this work was recognized later on by a conference of the Royal Society on quantum catalysis in enzymes in $2005 .{ }^{24}$ During the valley of $1967-1968$, Chance and coworkers identified oxidized flavoproteins (Fp including FAD) as another source of intrinsic fluorescence from mitochondria, ${ }^{25-27}$ which contributed to another rebound of publications in the following years.

The 3rd broad period of Chance publication curve from 1969-1977 appears to have the 2nd largest publication peak with studies ranging from NADH, flavoproteins, cytochoromes, calcium uptake to hydrogen peroxide generation. The use of oxidized flavoproteins together with NADH makes the in vivo measurement of redox status (Fp/NADH or $\mathrm{Fp} /$ $(\mathrm{Fp}+\mathrm{NADH}))$ possible by the fluorescence spectroscopy and imaging. Therefore Chance et al. developed this idea and technologies into translational researches by inventing NADH fluorometery, flying spot technology and cryogenic NADH/Fp redox scanning, etc. Still now, these are the only fluorescence signals used clinically, mainly for cancer detection. Chance wrote in his unpublished autobiography regarding the importance of his discovery of the fluorescence of NADH and Fp from mitochondria both in vivo and ex vivo: "... This was perhaps the most important discovery of my career because, for the first time, we could obtain optical signals from living mitochondrial tissues. A series of papers, exploring this discovery in the liver, kidney, adrenal gland, and brain, opened a new field of metabolic research...." Indeed, since 1950s Chance kept publishing on NADH and 
flavoproteins throughout the rest of his life. The work on NADH and flavoproteins, along with his other research work including the studies on cytochromes, ${ }^{28-30}$ discovery of the calcium uptake by mitochondria and its effects on bioenergetics, ${ }^{31}$ discovery of hydrogen peroxide generation by mitochondria, ${ }^{32}$ etc., really drove the publication of Chance onto a new level in the subsequent years until late 1970s, with about 20 papers annually.

The 4th period in 1978-1981 is only 4 years, and it is the beginning of three important new directions of publications. The first direction of new publications marked the beginning of Chance's effort in developing ${ }^{31} \mathrm{P}-\mathrm{NMR}$ techniques for bioenergetics studies in organs and human body. ${ }^{33-36}$ Chance funded himself to make an NMR machine for studying phosphorous energetics in human subjects including his own leg in vivo. ${ }^{33,37}$ The second new direction of publications was about the chemiluminescence ${ }^{38-40}$ emitted by the lipid peroxidation process in biological systems, which was the continuation of the interest of Chance on metabolism, redox reactions and free radicals. Chance published his studies on chemiluminescence from late 1970s to mid 1990s. He and coworkers probably provided one of the early experimental evidences for the generation of singlet oxygen in biological processes. $^{41-43}$ The third source of new publications was the studies ${ }^{44-48}$ by Chance and Power et al. using synchrotron radiation X-ray spectroscopy to illustrate structure-function relation for biomolecules including cytochrome oxidase, myoglobin, glyoxalase, etc.

The 5th period of Chance publication (19821989) has the highest peak in 1987 with an annual publication of 32 papers. That was on average two papers per three week at the age of 74 . That was the period when Chance and Leigh et al. had made significant progress in developing in vivo NMR for bioenergetics and applying it to diseases. From 1984 to 1987 , every year Chance and Leigh coauthored 10-15 papers according to the ISI Science Citation indexes. Other sources of contributions include the chemiluminescence studies and the synchrotron radiation studies aforementioned. After the peak in 1987, Chance publication lowered to 15 in 1990. In 1988, Chance and Leigh et al. initiated timeresolved measurements on hemoglobin and myoglobin in tissues. ${ }^{49,50}$ Chance then published with M. S. Patterson and B. C. Wilson the work on timeresolved optical spectroscopy, ${ }^{51}$ which made it possible to measure scattering and absorption coefficients in biological tissue. This paper was revolutionary for optical fields because it gave birth to the diffusive biomedical photonics.

The last two Gaussian periods of Chance publication (1990-1999 and 2000-2010) are truly optical and more translational periods of Chance's work. Chance, as a founding father of modern biophotonics, had collaborated with A. Yodh and a number of researchers since 1990s to develop NIR spectroscopy and imaging, including time resolved spectroscopy, photon diffusion tomography (PDT), phased array and their biomedical applications to study brain (fNIR), muscle and various diseases such as breast cancer. ${ }^{52-63}$ Chance et al. also integrated NIR spectroscopy/imaging with magnetic resonance imaging. ${ }^{58,64}$ Since 2002, Chance had publications with Zheng and Glickson et al. on molecular beacons, nanoparticles and photodynamic therapy. ${ }^{65-69}$ His publication rebounded in 1991 and maintained an average publication of about 18 papers annually for 15 years until 2005. In 2006, Chance ran out of his research funding and since then he was invited to teach and conduct research in Singapore, Mainland China and Taiwan half of the time each year, while $\mathrm{Li}$ and $\mathrm{Xu}$ were maintaining his redox scanning lab at the University of Pennsylvania. His publication went down but was still maintained at about eight papers on average per year until 2010. During this period of time, he and his coworkers investigated on the development of optical imaging biomarkers for cancer diagnosis and tumor metastatic potential, ${ }^{70-73}$ stem cell stemness ${ }^{74}$ and a metabolometer $^{75}$ for monitoring human nutritional status. Chance worked on research till the last few days of his life. Li et al. remembered discussing research data with him in the hospital room three days before his passing away.

Citation trends. The annual citations of the Chance publications shown in Fig. 2 exhibited 4 rising $(\mathrm{R})$ phases and 2 leveling $(\mathrm{L})$ phases. The 1st rising phase (R1) from 1947 till 1958, increasing with about 20 citations per year, is presumably based on his studies on enzyme kinetics and pioneering work on oxidative phosphorylation. The phase R2 from 1959 to 1972 has the steepest slope ( $\sim 60$ citations per year) among the four $\mathrm{R}$ phases, indicating his scientific influence expanded at the fastest pace during these years. This expansion of fame should be significantly contributed by his 


\section{Z. Li et al.}

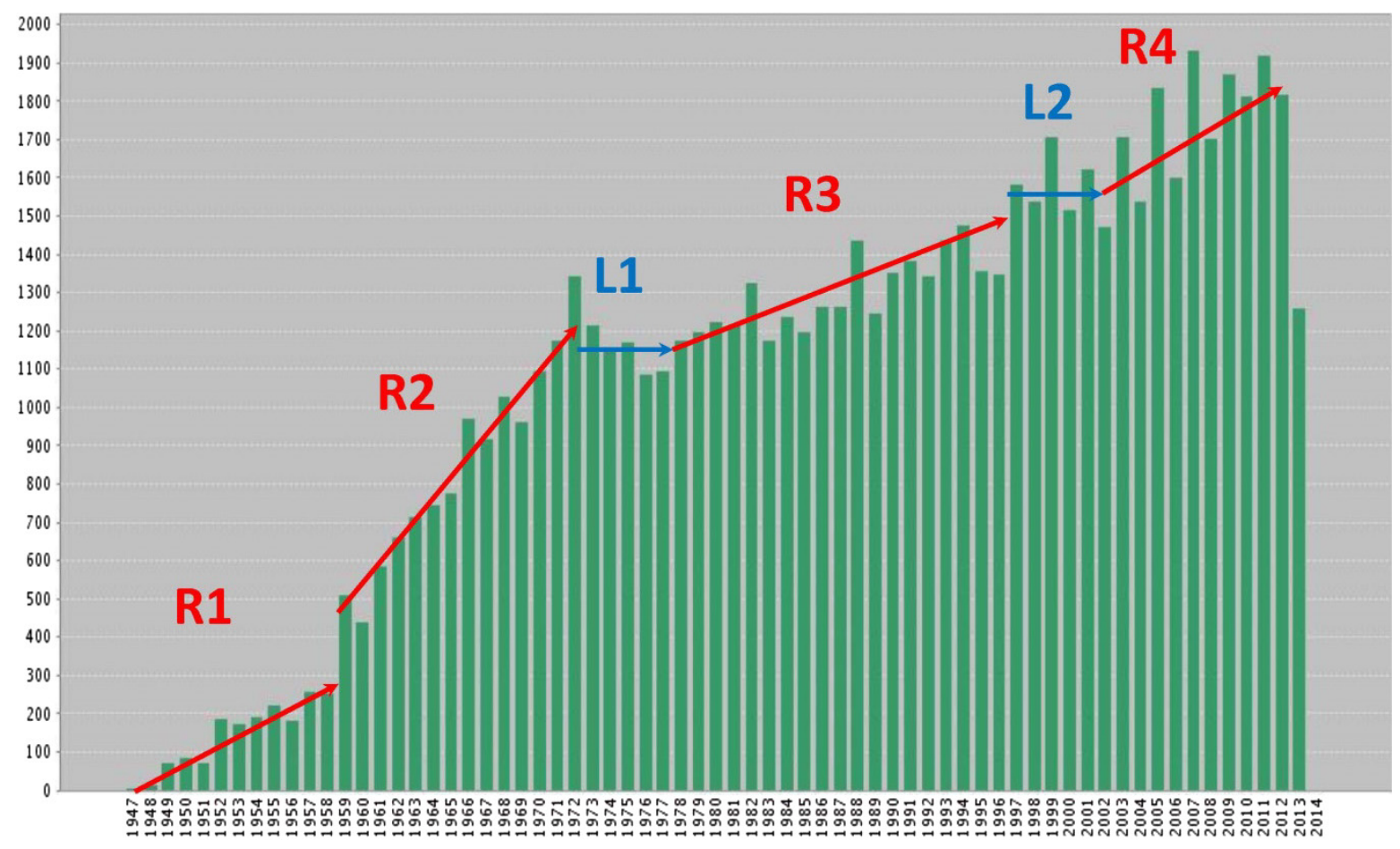

Fig. 2. The annual citations of publications of Britton Chance from 1947-2013. Graph reproduced from ISI Citation Indexes (accessed in April, 2013 excluding meeting abstracts) with modifications. R: rising phase; L: leveling phase as described in the text.

previous work on the stop-flow measurement of enzyme-substrate kinetics. Indeed, "when Manfred Eigen won the [Nobel] prize in 1967 for his temperature jump method that could dissect chemical reactions even more finely than Brit's stopped flow approach, he publicly regretted not being able to share the honor with Britton Chance" (Gottfried Schatz, 2011 http://www.med.upenn.edu/biocbiop/ chance/symposium/schatz/schatz_talk.html). For Britton Chance, this was also a period with important new discoveries that were nothing short of importance compared to his first experimental demonstration of the existence of Michaelis-Menten enzyme-substrate complex. He and his coworkers developed the dual-beam spectrometer for biological studies and characterized the electron-transport chain and bioenergetic process in mitochondrial oxidative phosphorylation. They discovered the fluorescence of $\mathrm{NADH}$ and flavoproteins from mitochondria and translated these methods to tissue in vivo with many clinical applications. They also discovered the electron-tunneling in biological systems, and identified the calcium uptake and hydrogen peroxide generation by mitochondria. In 1974, Britton Chance was awarded the US National Medal of Sciences. The first leveling phase L1 corresponds to
1973-1976. The third rising R3 phase from 1976 to 1996 includes the original development of phosphorous NMR and diffusive optical spectroscopy and imaging methods, with a slope similar to that of first phase, followed by the leveling phase L2 from 1997-2001 with over 1500 citations per year on average. The rising phase R4 is from 2002 to 2012 with a slope of 30 citations per year higher than that of R1 and R3.

HistCite analysis. Figures 3 and 4 show the citation analysis results from HistCite ${ }^{\mathrm{TM}}$ for top 30 papers with the highest global citations and local citations, respectively. The local citations refer to the citations only by the papers published by Chance himself. The citation relationships among these papers are displayed in these two figures. The detailed paper information and the number of citations are also listed in the order of publication years from the oldest to the latest.

For global citation analysis, there are six papers cited by over 1000 times. The No. 1 highest cited paper $^{76}$ is a review by Chance et al. in 1979 on hydrogen peroxide metabolism in mammalian organs, with nearly 4000 citations. The fourth highest cited paper ${ }^{77}$ is also about hydrogen peroxide, i.e., its generation by mitochondria, general 


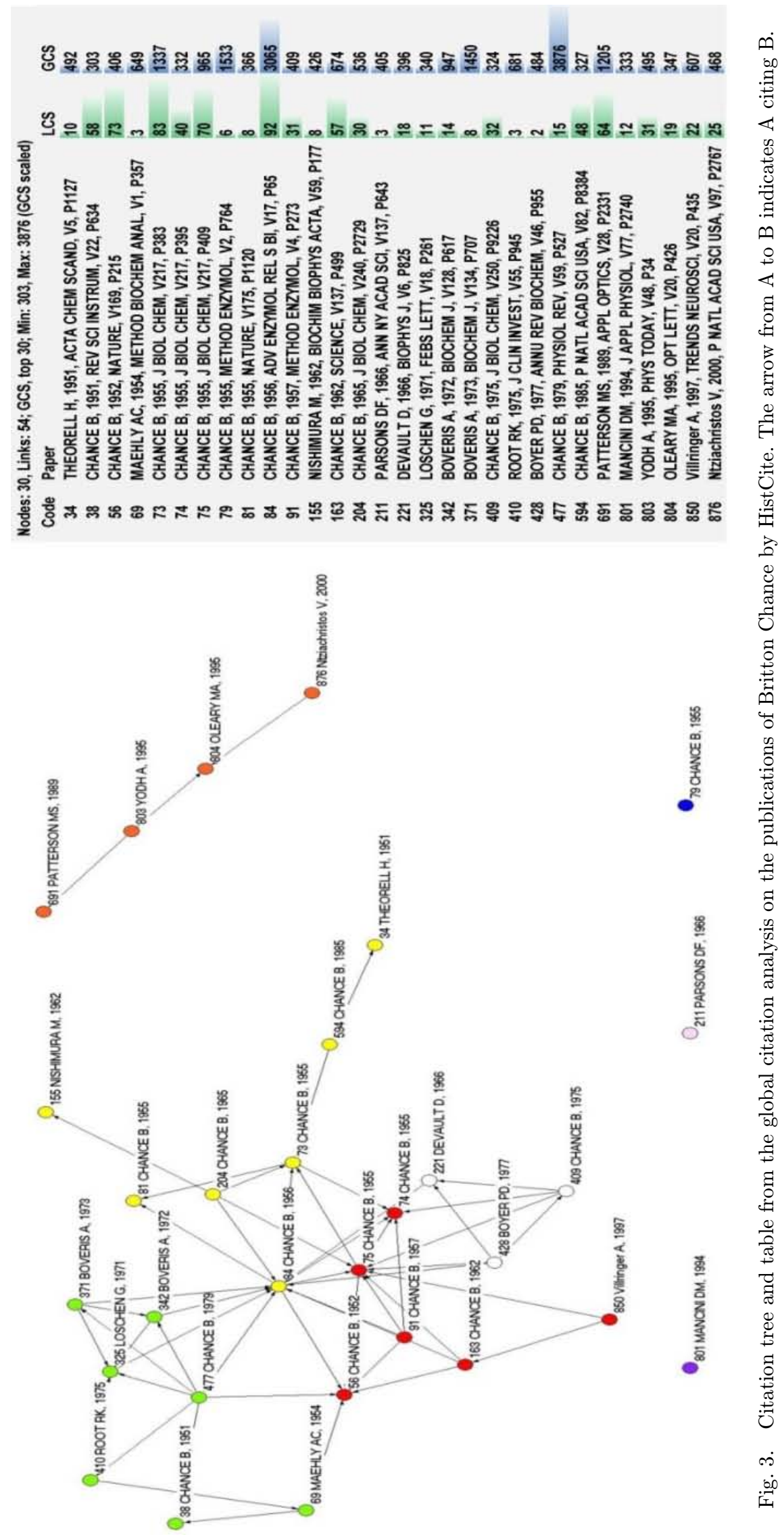




\section{Z. Li et al.}
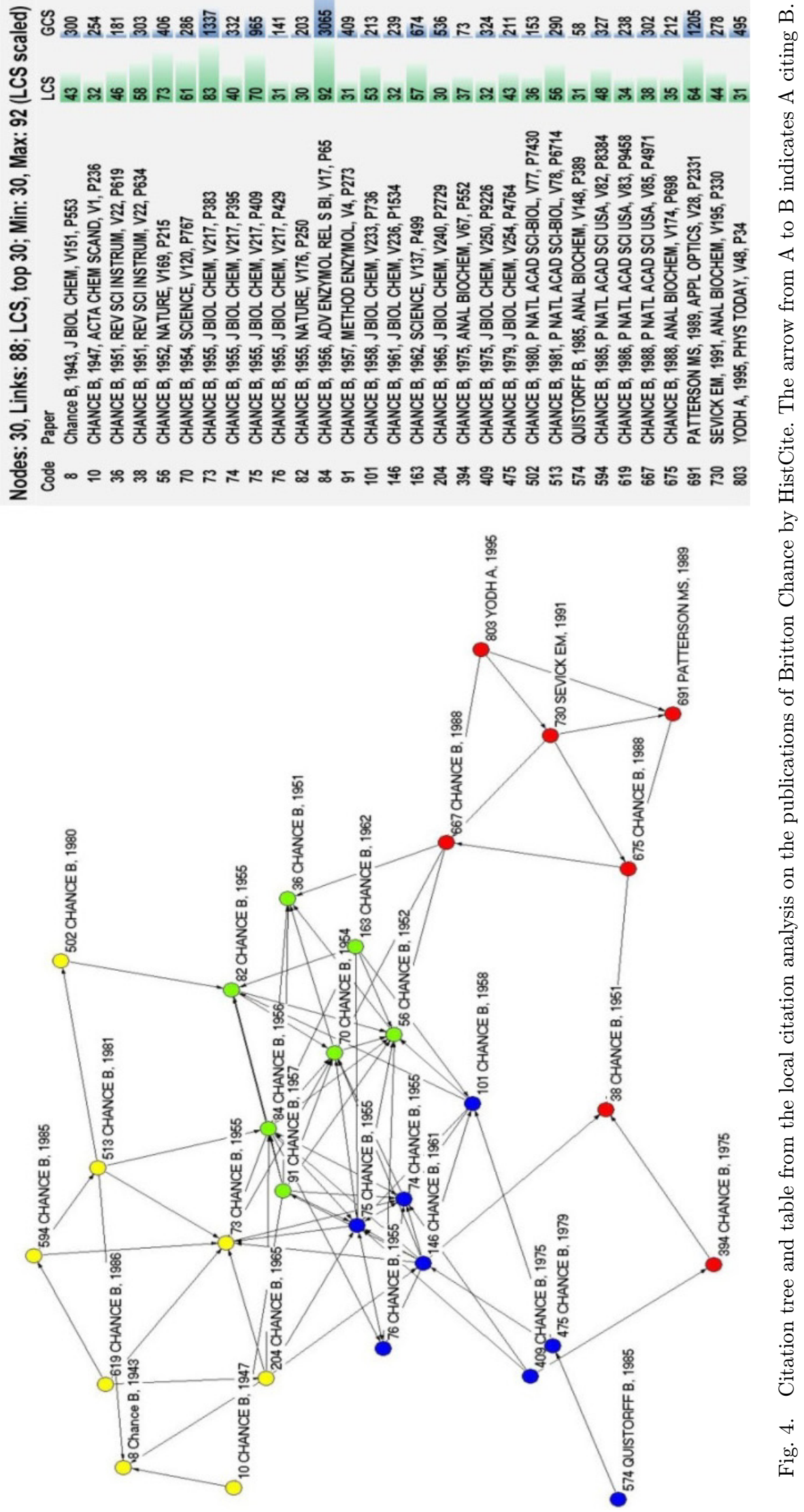


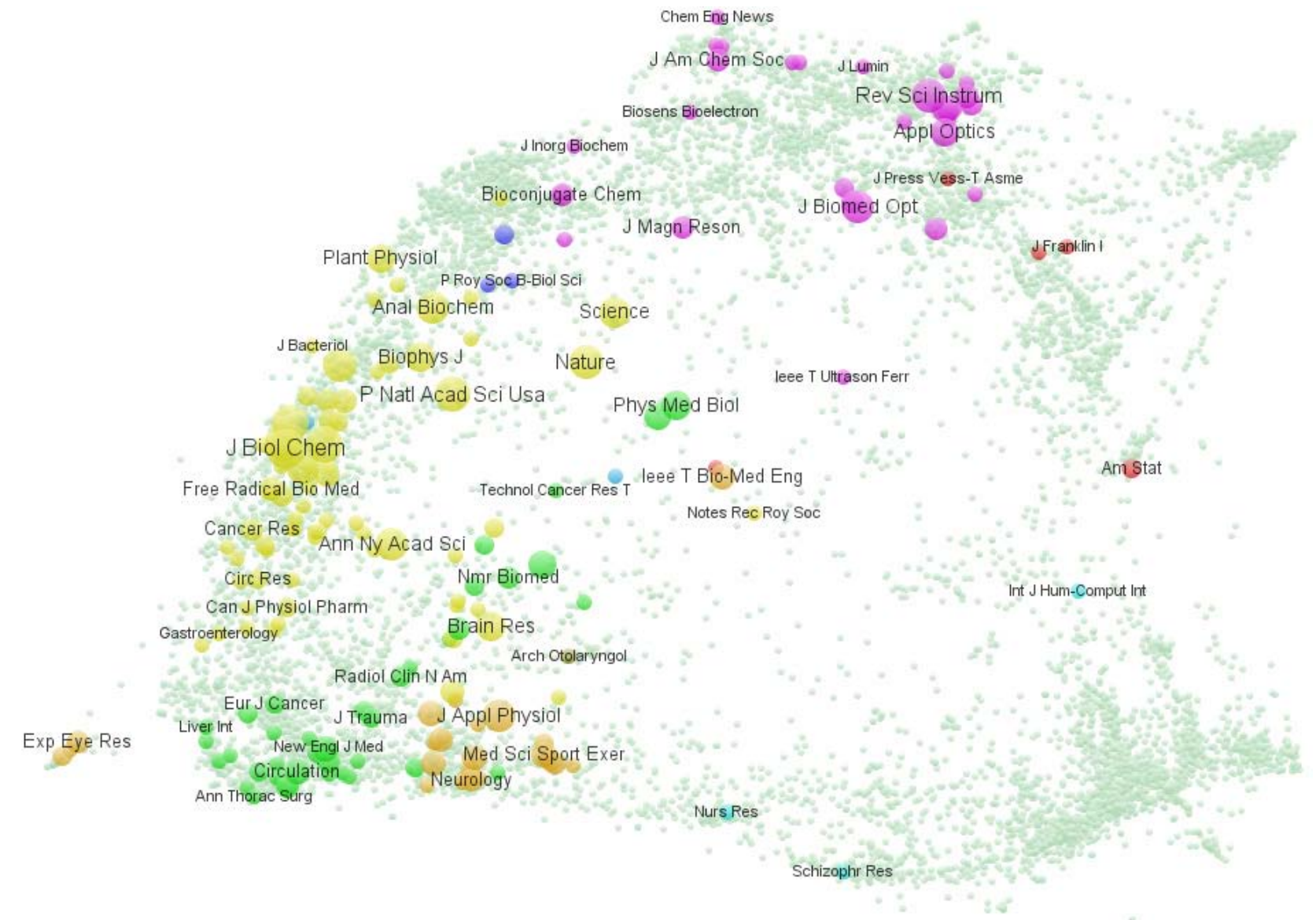

Fig. 5. Journal maps of Britton Chance publications. The size of sphere is proportional to number of publications.

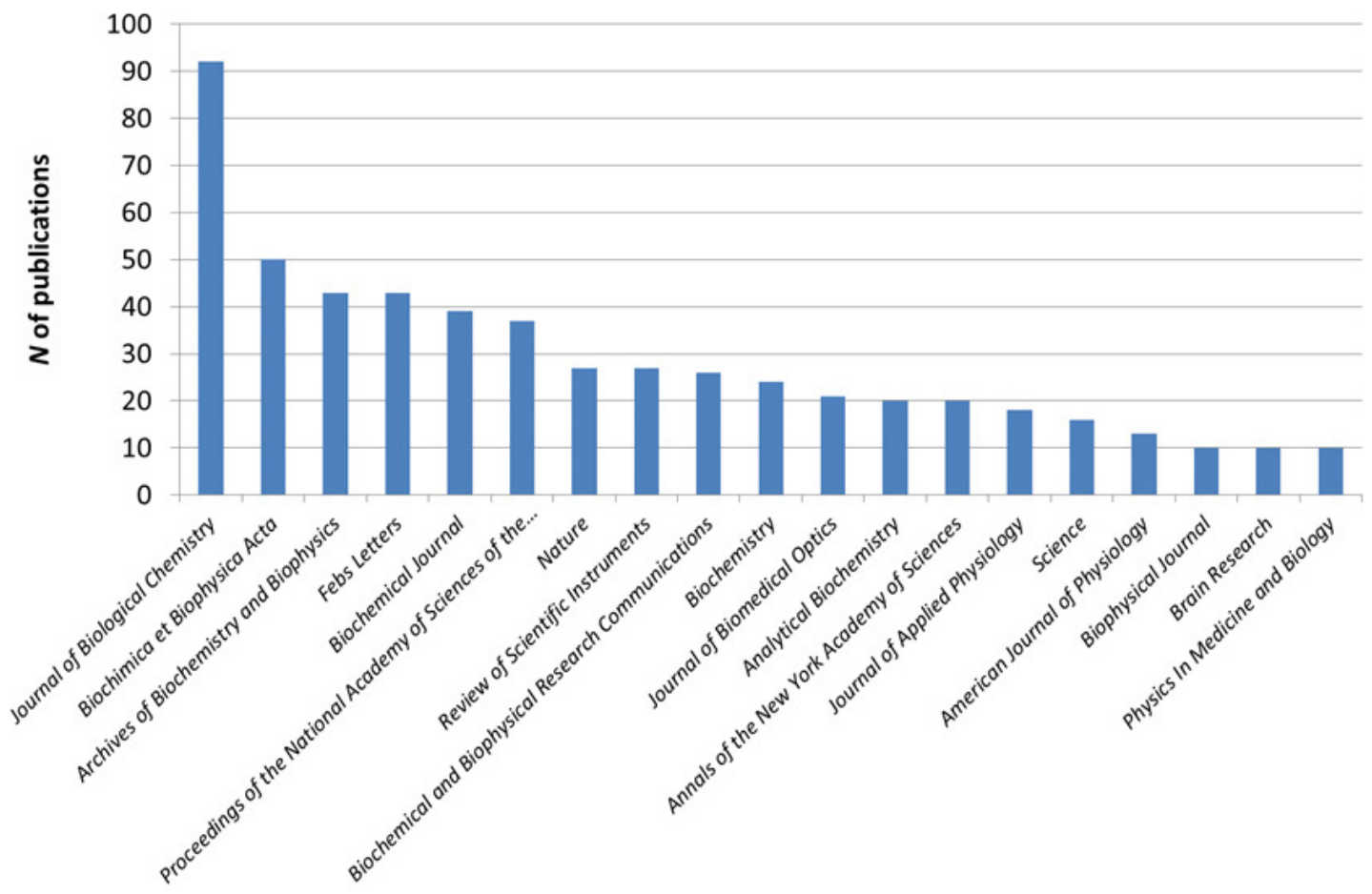

Fig. 6. Top journals with most publications from Britton Chance. 


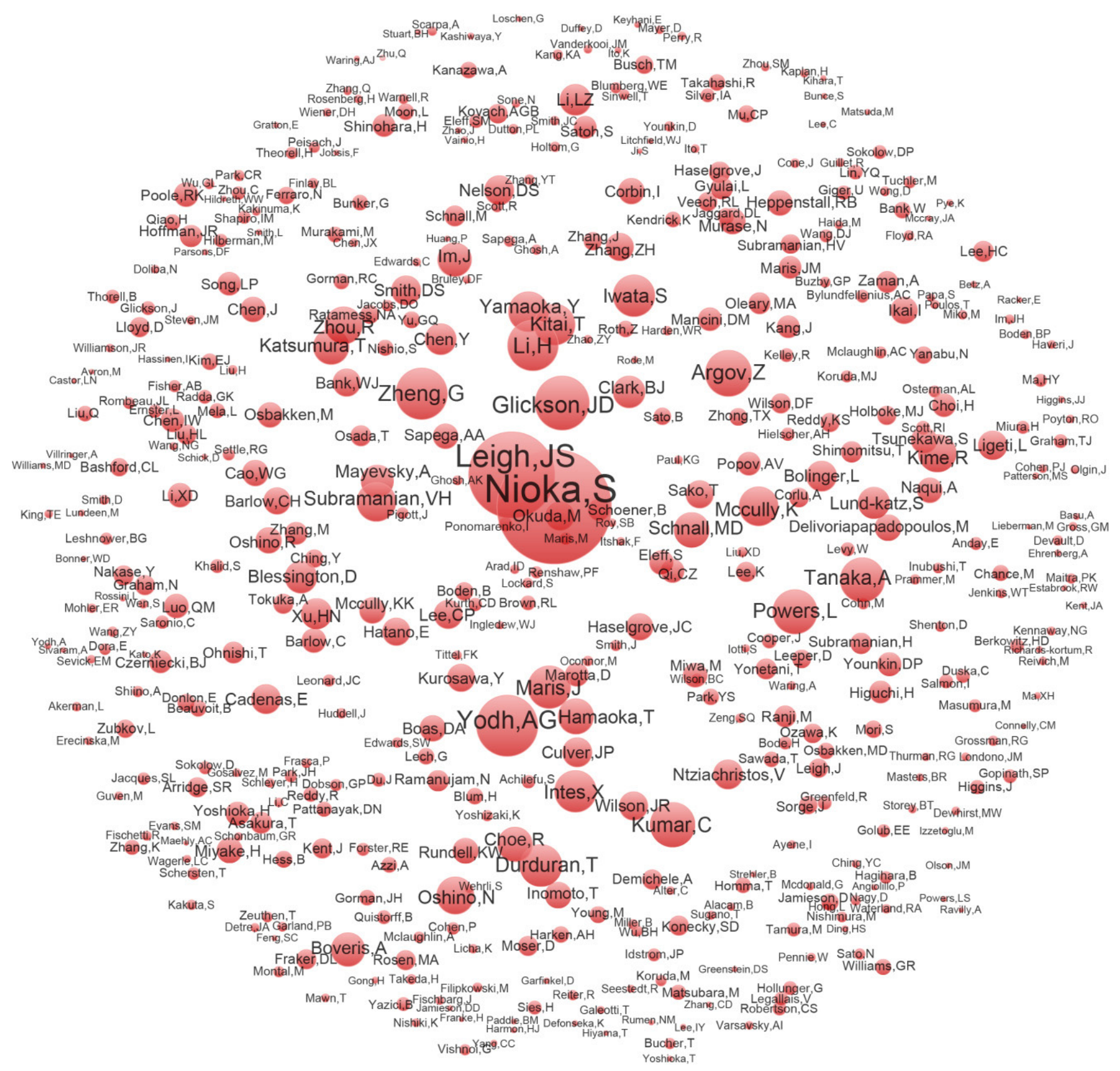

Fig. 7. Coauthor maps. All coauthors with more than 1 publication with Britton Chance. The size of sphere is proportional to the number of papers.

properties and effects. The second ${ }^{78}$ and fifth ${ }^{8}$ highest cited papers are both about mitochondrial respiratory chain and oxidative phosphorylation. The third highest cited paper ${ }^{79}$ is about assays of catalases and peroxidases. The sixth highest cited paper ${ }^{51}$ is about the time-resolved optical spectroscopy marking the beginning of photon-diffusion spectroscopy and imaging. Among the top 30 globally most cited papers, 4 are about enzyme assay and kinetic studies, 1 about dual beam spectrometer, 13 about mitochondrial oxidative phosphorylation, 5 about NIR imaging and spectroscopy (biophotonics), 1 about in vivo ${ }^{31} \mathrm{P}-\mathrm{NMR}, 1$ about electron tunneling, 1 about calcium ion uptake by mitochondrion, 5 about hydrogen peroxide.

For local citation analysis, three of the top six highest globally cited papers remain in the top six. They are two papers on respiratory chain/oxidative phosphorylation $^{8,78}$ and one on time-resolved optical spectroscopy. ${ }^{51}$ The other three ranked within 
the top six locally cited are all on respiratory chain/ oxidative phosphorylation. ${ }^{10,80,81}$ Among the top 30 locally most cited papers, 4 papers are about enzyme assay and kinetic studies, 1 about dual beam spectrometer, 16 about mitochondrial respiration and redox state, 1 about calcium ion uptake by mitochondrion, 5 about NIR imaging and spectroscopy (biophotonics), 4 about in vivo ${ }^{31} \mathrm{P}-\mathrm{NMR}$. It appears that the studies on mitochondrial metabolism and the related development of optical techniques are most-often cited by Chance himself. From the LCS map (Fig. 4) we can see that these work form the center of the citation tree, with biophotonics and NMR bioenergetics as side lobes linked to the center. This relationship indicates mitochondrial metabolism as a central basis of Chance research rationale and a long-term pursued research goal. During the last 20 years of his life, Chance appeared to have diversified his research and moved away from this research focus by developing new techniques such as in vivo NIR spectroscopy and imaging. Nevertheless, these new methods, although with many novel applications beyond mitochondrial metabolism, are important for probing and understanding about tissue metabolism.

Diversity of research fields and collaborators. Figure 5 shows the journal maps of Chance publications. The size of sphere is proportional to the logarithm of the number of publications. Among the 266 journals, Chance has published in this ISI database, the leading journals include Journal of Biological Chemistry, Proceedings of National Academy of Sciences (PNAS), Nature, Review of Scientific Instrumentation, Journal Biomedical Optics, Science, Journal of Applied Physiology, Analytical Biochemistry, New England Journal of Medicine, Physics in Medicine and Biology, Brain Research, Medicine \& Science in Sports \& Exercise, NMR in Biomedicine, and so on. From these journal titles (only a portion of journal titles shown in Fig. 5), we can see that the research of Chance covered multiple disciplines ranging from physics, engineering, biology to medicine. Figure 6 provides more detailed information about the top 19 journals with most publications from Chance. Impressively there are 92 papers in Journal of Biological Chemistry, 37 in PNAS, 27 in Nature and 16 in Science. Figure 7 is the coauthor map showing all coauthors with more than 1 publication with Chance. The size of sphere is proportional to the number of papers. We can see S. Nioka, J. S. Leigh, A. Yodh as the top
3 coauthors with the most coauthored papers with Britton Chance.

\section{Discussion and Summary}

Note that all the above analysis and discussion are based on the 1023 records collected by ISI citation database in April, 2013. Our own collection of Britton Chance's publication record, which is still an ongoing process, showed over 1500 manuscripts. We may perform additional analysis on this database once it is complete. This may provide a more complete picture about his research activity. Furthermore, considering the tremendously diversified multi-disciplinary research conducted by Britton Chance, we can only cover some major research topics we are familiar with. The scientometric methods we used have a limited number of angles of perspectives. It is highly possible we fail to disclose many interesting or meaningful connections among the research publications by Chance and his research activities.

In summary, we have presented the first scientometric analysis on a world preeminent scholar Britton Chance, whose research spanned multiple disciplines from physics, engineering, biology to medicine. We try to understand the profiles of his annual publications and citations by correlating them with his research activities. We have also summarized some key research areas that Britton Chance had focused and exerted great impact on. Among the diversified research studies Chance had conducted, metabolism and the development of physical methods to probe it appeared to be the central thread that connected all the dots. We also note that Chance had many excellent collaborators from very diversified fields of science throughout his life, and that had enabled him to make such a great amount of publications with tremendous impact on science.

\section{References}

1. L. Z. Li, S. Nioka, K. A. Kang, "Dedication: Britton Chance, M.D., Ph.D., D.Sc.," Adv. Exp. Med. Biol. 765, v-xiv (2013).

2. L. Leydesdorff, "Eugene Garfield and algorithmic historiography: Co-words, co-authors, and journal names," Ann Library Inform. Stud. 57, 248-260 (2010). 
3. B. Chance, J. Brainerd, F. Cajori, G. Millikan, "The kinetics of the enzyme-substrate compound of peroxidase and their relation to the Michaelis theory," Science 92, 455 (1940).

4. B. Chance, "The kinteics of the enzyme-substrate compound of peroxidase," J. Biol. Chem. 151, 553577 (1943).

5. H. Theorell, B. Chance, "Studies on liver alcohol dehydrogenase. II. The kinetics of the compound of horse liver alcohol dehydrogenase and reduced diphosphopyridine nucleotide," Acta Chem. Scand. 5, 1127-1144 (1951).

6. B. Chance, "Rapid and sensitive spectrophotometry. III. A double beam apparatus," Rev. Sci. Instrum. 22, 634-638 (1951).

7. B. Chance, "An intermediate compound in the catalase-hydrogen peroxide reaction," Acta Chem. Scand. 1, 236-267 (1947).

8. B. Chance, G. R. Williams, "Respiratory enzymes in oxidative phosphorylation. I. Kinetics of oxygen utilization," J. Biol. Chem. 217, 383-393 (1955).

9. B. Chance, G. R. Williams, "Respiratory enzymes in oxidative phosphorylation. II. Difference spectra," J. Biol. Chem. 217, 395-407 (1955).

10. B. Chance, G. R. Williams, "Respiratory enzymes in oxidative phosphorylation. III. The steady state," J. Biol. Chem. 217, 409-427 (1955).

11. B. Chance, G. R. Williams, "Respiratory enzymes in oxidative phosphorylation. IV. The respiratory chain," J. Biol. Chem. 217, 429-438 (1955).

12. B. Chance, B. Hess, "Metabolic control mechanisms. IV. The effect of glucose upon the steady state of respiratory enzymes in the ascites cell," J. Biol. Chem. 234, 2421-2427 (1959).

13. B. Chance, B. Hess, "Metabolic control mechanisms. III. Kinetics of oxygen utilization in ascites tumor cells," J. Biol. Chem. 234, 2416-2420 (1959).

14. B. Chance, B. Hess, "Metabolic control mechanisms. II. Crossover phenomena in mitochondria of ascites tumor cells," J. Biol. Chem. 234, 2413-2415 (1959).

15. B. Chance, B. Hess, "Metabolic control mechanisms. I. Electron transfer in the mammalian cell," J. Biol. Chem. 234, 2404-2412 (1959).

16. B. Chance, D. Garfinkel, J. Higgins, B. Hess, "Metabolic control mechanisms. V. A solution for the equations representing interaction between glycolysis and respiration in ascites tumor cells," J. Biol. Chem. 235, 2426-2439 (1960).

17. B. Hess, B. Chance, "Metabolic control mechanisms. VI. chemical events after glucose addition to ascites tumor cells," J. Biol. Chem. 236, 239-246 (1961).

18. B. Chance, H. Baltscheffsky, "Respiratory enzymes in oxidative phosphorylation. VII. Binding of intramitochondrial reduced pyridine nucleotide," J. Biol. Chem. 233, 736-739 (1958).
19. B. Chance, F. Jobsis, "Changes in fluorescence in a frog sartorius muscle following a twitch," Nature 184, 195-196 (1959).

20. B. Chance, B. Schoener, P. Cohen, F. Jobsis, "Localized fluorometry of oxidation-reduction states of intracellular pyridine nucleotide in brain and kidney cortex of anesthetized rat," Science 136, 325 (1962).

21. B. Chance, P. Cohen, F. Jobsis, B. Schoener, "Intracellular oxidation-reduction states in vivo," Science 137, 499-508 (1962).

22. B. Chance, B. Schoener, "Correlation of oxidationreduction changes of intracellular reduced pyridine nucleotide and changes in electroencephalogram of the rat in anoxia," Nature 195, 956-958 (1962).

23. D. DeVault, B. Chance, "Studies of photosynthesis using a pulsed laser. I. Temperature dependence of cytochrome oxidation rate in chromatium. Evidence for tunneling," Biophys. J. 6, 825-847 (1966).

24. N. S. Scrutton, M. J. Sutcliffe, P. Leslie Dutton, "Quantum catalysis in enzymes: Beyond the transition state theory paradigm. A Discussion Meeting held at the Royal Society on 14 and 15 November 2005," J. R. Soc. Interface / the R. Soc. 3, 465-469 (2006).

25. B. Chance, L. Ernster, P. B. Garland, C. P. Lee, P. A. Light, T. Ohnishi, C. I. Ragan, D. Wong, "Flavoproteins of the mitochondrial respiratory chain," Proc. Natl. Acad. Sci. USA 57, 1498-1505 (1967).

26. P. B. Garland, B. Chance, L. Ernster, C. P. Lee, D. Wong, "Flavoproteins of mitochondrial fatty acid oxidation," Proc. Natl. Acad. Sci. USA 58, 16961702 (1967).

27. I. Hassinen, B. Chance, "Oxidation-reduction properties of the mitochondrial flavoprotein chain," Biochem. Biophys. Res. Commun. 31, 895-900 (1968).

28. M. V. Gilmour, M. R. Lemberg, B. Chance, "Cytochrome oxidase and its derivatives. IX. Spectrophotometric studies on the rapid reaction of ferrous cytochrome c oxidase with molecular oxygen under conditions of complete and partial oxygenation," BBA - Bioenergetics 172, 37-51 (1969).

29. B. Chance, "Cytochromes: Chemical and structural aspects," Science 159, 654-658 (1968).

30. B. Chance, C. Saronio, J. S. Leigh Jr, "Functional intermediates in the reaction of membrane bound cytochrome oxidase with oxygen," J. Biol. Chem. 250, 9226-9237 (1975).

31. B. Chance, "The energy-linked reaction of calcium with mitochondria," J. Biol. Chem. 240, 2729-2748 (1965).

32. G. Loschen, L. Flohé, B. Chance, "Respiratory chain linked $\mathrm{H}_{2} \mathrm{O}_{2}$ production in pigeon heart mitochondria," FEBS Lett. 18, 261-264 (1971). 
33. B. Chance, S. Eleff, J. S. Leigh, Jr., "Noninvasive, nondestructive approaches to cell bioenergetics," Proc. Natl. Acad. Sci. USA 77, 7430-7434 (1980).

34. P. B. Garlick, G. K. Radda, P. J. Seeley, B. Chance, "Phosphorus NMR studies on perfused heart," Biochem. Biophy. Res. Commun. 74, 1256-1262 (1977).

35. B. Chance, G. Radda, P. J. Seeley, I. Silver, Y. Nakase, M. Bond, G. McDonald, "31P NMR of excised and in situ brain tissues," in NMR and Biochemistry: A Symposium Honoring Mildred Cohn, S. Opella \& P. Lu, Eds., pp. 269-281, Marcel Dekker, Inc., New York (1979).

36. A. C. McLaughlin, H. Takeda, B. Chance, "Rapid ATP assays in perfused mouse liver by 31P NMR," Proc. Natl. Acad. Sci. USA 76, 5445-5449 (1979).

37. J. Cohen, "Scientists who fund themselves," Science 279, 178-181 (1998).

38. A. Boveris, B. Chance et al., "Enhancement of the chemiluminescence of perfused rat liver and of isolated mitochondria and microsomes by hydroperoxides," Adv. Exp. Med. Biol. 2, 975-984 (1978).

39. A. Boveris, E. Cadenas, R. Reiter, M. Filipkowski, Y. Nakase, B. Chance, "Organ chemiluminescence: Noninvasive assay for oxidative radical reactions," Proc. Natl. Acad. Sci. USA 77, 347-351 (1980).

40. E. Cadenas, A. I. Varsavsky, A. Boveris, B. Chance, "Low level chemiluminescence of the cytochrome c-catalyzed decomposition of hydrogen peroxide," FEBS Lett. 113, 141-144 (1980).

41. E. Cadenas, R. P. Daniele, B. Chance, "Low level chemiluminescence of alveolar macrophages: Spectral evidence for singlet oxygen generation," FEBS Lett. 123, 225-228 (1981).

42. A. Greer, "Christopher Foote's discovery of the role of singlet oxygen $\left[{ }^{1} \mathrm{O}_{2}\left({ }^{1} \Delta_{\mathrm{g}}\right)\right]$ in photosensitized oxidation reactions," Acc. Chem. Res. 39, 797-804 (2006).

43. M. J. Thomas, P. S. Shirley, C. C. Hedrick, L. R. DeChatelet, "Role of free radical processes in stimulated human polymorphonuclear leukocytes", Biochemistry 25, 8042-8048 (1986).

44. L. Powers, W. E. Blumberg, B. Chance, C. H. Barlow, J. Leigh, J. S., J. Smith, T. Yonetani, S. Vik, "Structure and function of copper atoms in cytochrome oxidase," Adv. Exp. Med. Biol. 2, 863871 (1978).

45. L. Powers, W. E. Blumberg, B. Chance, C. H. Barlow, J. S. Leigh, Jr., J. Smith, T. Yonetani, S. Vik, J. Peisach, "The nature of the copper atoms of cytochrome c oxidase as studied by optical and x-ray absorption edge spectroscopy," Biochim. Biophys. Acta 546, 520-538 (1979).

46. J. Peisach, L. Powers, W. E. Blumberg, B. Chance, "Stellacyanin. Studies of the metal-binding site using x-ray absorption spectroscopy," Biophys. J. 38, 277-285 (1982).

47. B. Chance, R. Fischetti, L. Powers, "Structure and kinetics of the photoproduct of carboxymyoglobin at low temperatures: An X-ray absorption study," Biochemistry 22, 3820-3829 (1983).

48. L. Garcia-Iniguez, L. Powers, B. Chance, S. Sellin, B. Mannervik, A. S. Mildvan, "X-ray absorption studies of the $\mathrm{Zn}^{2+}$ site of glyoxalase I," Biochemistry 23, 685-689 (1984).

49. B. Chance, J. S. Leigh, H. Miyake, D. S. Smith, S. Nioka, R. Greenfeld, M. Finander, K. Kaufmann, W. Levy, M. Young, "Comparison of time-resolved and -unresolved measurements of deoxyhemoglobin in brain," Proc. Natl. Acad. Sci. USA 85, 4971-4975 (1988).

50. B. Chance, S. Nioka, J. Kent, K. McCully, M. Fountain, R. Greenfeld, G. Holtom, "Time-resolved spectroscopy of hemoglobin and myoglobin in resting and ischemic muscle," Anal. Biochem. 174, 698707 (1988).

51. M. S. Patterson, B. Chance, B. C. Wilson, "Time resolved reflectance and transmittance for the noninvasive measurement of tissue optical properties," Appl. Opt. 28, 2331-2336 (1989).

52. M. A. O'Leary, D. A. Boas, B. Chance, A. G. Yodh, "Refraction of diffuse photon density waves," Phys. Rev. Lett. 69, 2658-2661 (1992).

53. D. A. Boas, M. A. O'Leary, B. Chance, A. G. Yodh, "Scattering of diffuse photon density waves by spherical inhomogeneities within turbid media: Analytic solution and applications," Proc. Natl. Acad. Sci. USA 91, 4887-4891 (1994).

54. X. Li, B. Beauvoit, R. White, S. Nioka, B. Chance, A. G. Yodh, Optical Tomography, Photon Migration, and Spectroscopy of Tissue and Model Media: Theory, Human Studies, and Instrumentation Tumor Localization Using Fluorescence of Indocyanine Green(ICG) in Rat Models, Vol. 789 SPIE, San Jose, CA, USA (1995).

55. A. Yodh, B. Chance, "Spectroscopy and imaging with diffusing light," Phys. Today 48, 34-40 (1995).

56. X. D. Li, T. Durduran, A. G. Yodh, B. Chance, D. N. Pattanayak, "Diffraction tomography for biochemical imaging with diffuse-photon density waves," Opt. Lett. 22, 573-575 (1997).

57. V. Ntziachristos, X. Ma, A. G. Yodh, B. Chance, OSA Trends in Optics and Photonics, E. M. SevickMuraca, J. A. Izatt, M. N. Ediger, Eds., Quantitation of Functional Motor Cortex Activity using Time-Resolved Spatially Localized NIR Spectroscopy, pp. 200-204, Optical Society of America, Washington, DC (1998).

58. V. Ntziachristos, A. G. Yodh, M. D. Schnall, B. Chance, "MRI-guided diffuse optical spectroscopy 
of malignant and benign breast lesions," Neoplasia 4, 347-354 (2002).

59. G. Yu, T. Durduran, G. Lech, C. Zhou, B. Chance, E. R. Mohler, 3rd, A. G. Yodh, "Time-dependent blood flow and oxygenation in human skeletal muscles measured with noninvasive near-infrared diffuse optical spectroscopies," J. Biomed. Opt. 10, 024027 (2005).

60. X. Intes, B. Chance, M. J. Holboke, A. G. Yodh, "Interfering diffusive photon-density waves with an absorbing-fluorescent inhomogeneity," Opt. Express 8, 223-231 (2001).

61. A. Villringer, B. Chance, "Non-invasive optical spectroscopy and imaging of human brain function," Trends Neurosci. 20, 435-442 (1997).

62. D. M. Mancini, L. Bolinger, H. Li, K. Kendrick, B. Chance, J. R. Wilson, "Validation of near-infrared spectroscopy in humans," J. Appl. Physiol. 77, 2740-2747 (1994).

63. E. M. Sevick, B. Chance, J. Leigh, S. Nioka, M. Maris, "Quantitation of time- and frequency-resolved optical spectra for the determination of tissue oxygenation," Anal. Biochem. 195, 330-351 (1991).

64. V. Ntziachristos, A. G. Yodh, M. Schnall, B. Chance, "Concurrent MRI and diffuse optical tomography of breast after indocyanine green enhancement," Proc. Natl. Acad. Sci. USA 97, 2767-2772 (2000).

65. G. Zheng, H. Li, K. Yang, D. Blessington, K. Licha, S. Lund-Katz, B. Chance, J. D. Glickson, "Tricarbocyanine cholesteryl laurates labeled LDL: New near infrared fluorescent probes (NIRFs) for monitoring tumors and gene therapy of familial hypercholesterolemia," Bioorg. Med. Chem. Lett. 12, 1485-1488 (2002).

66. M. Zhang, Z. Zhang, D. Blessington, H. Li, T. M. Busch, V. Madrak, J. Miles, B. Chance, J. D. Glickson, G. Zheng, "Pyropheophorbide 2-deoxyglucosamide: A new photosensitizer targeting glucose transporters," Bioconjug. Chem. 14, 709-714 (2003).

67. J. Chen, K. Stefflova, M. J. Niedre, B. C. Wilson, B. Chance, J. D. Glickson, G. Zheng, "Protease-triggered photosensitizing beacon based on singlet oxygen quenching and activation," J. Am. Chem. Soc. 126, 11450-11451 (2004).

68. Z. Zhang, D. Blessington, H. Li, T. M. Busch, J. Glickson, Q. Luo, B. Chance, G. Zheng, "Redox ratio of mitochondria as an indicator for the response of photodynamic therapy," J. Biomed. Opt. 9, 772-778 (2004).

69. Z. Zhang, H. Li, Q. Liu, L. Zhou, M. Zhang, Q. Luo, J. Glickson, B. Chance, G. Zheng, "Metabolic imaging of tumors using intrinsic and extrinsic fluorescent markers," Biosens. Bioelectron. 20, 643650 (2004).

70. L. Z. Li, R. Zhou, H. N. Xu, L. Moon, T. Zhong, E. J. Kim, H. Qiao, R. Reddy, D. Leeper, B. Chance, J. D. Glickson, "Quantitative magnetic resonance and optical imaging biomarkers of melanoma metastatic potential," Proc. Natl. Acad. Sci. USA 106, 66086613 (2009).

71. L. Z. J. Li, R. Zhou, T. Zhong, L. Moon, E. J. Kim, H. Qiao, S. Pickup, M. J. Hendrix, D. Leeper, B. Chance, J. D. Glickson, "Predicting melanoma metastatic potential by optical and magnetic resonance imaging," Adv. Exp. Med. Biol. 599, 67-78 (2007).

72. H. N. Xu, S. Nioka, J. D. Glickson, B. Chance, L. Z. $\mathrm{Li}$, "Quantitative mitochondrial redox imaging of breast cancer metastatic potential," J. Biomed. Opt. 15, 036010 (2010).

73. H. N. Xu, J. Tchou, B. Chance, L. Z. Li, "Imaging the redox states of human breast cancer core biopsies," Adv. Exp. Med. Biol. 765, 343-349 (2013).

74. H. N. Xu, R. C. Addis, D. F. Goings, S. Nioka, B. Chance, J. D. Gearhart, L. Z. Li, "Imaging redox state heterogeneity within individual embryonic stem cell colonies," J. Innov. Opt. Health Sci. 4, 279-288 (2011).

75. J.-R. Horng, S. Nioka, A. Quo, B. Chance, "A novel time-shared fluorometer gives the mitochondrial redox state as the ratio of two components of the respiratory chain of the animal and human buccal cavity with quantitative measures of the redox energy state," J. Innov. Opt. Health Sci. 3, 235-245 (2010).

76. B. Chance, H. Sies, A. Boveris, "Hydroperoxide metabolism in mammalian organs," Physiol. Rev. 59, 527-605 (1979).

77. A. Boveris, B. Chance, "The mitochondrial generation of hydrogen peroxide. General properties and effect of hyperbaric oxygen," Biochem. J. 134, 707716 (1973).

78. B. Chance, G. R. Williams, "The respiratory chain and oxidative phosphorylation," Adv. Enzymol. Relat. Subj. Biochem. 17, 65-134 (1956).

79. B. Chance, A. C. Maehly, "Assay of catalases and peroxidases," Methods Enzymol. 2, 764-775 (1955).

80. B. Chance, "Spectra and reaction kinetics of respiratory pigments of homogenized and intact cells," Nature 169, 215-221 (1952).

81. B. Chance, "Spectrophotometry of intracellular respiratory pigments," Science 120, 767-775 (1954). 